Supporting Information

\title{
High-performance borophene/graphene
}

\section{heterostructure anode of lithium-ion batteries}

\section{achieved via controlled interlayer spacing $\dagger$}

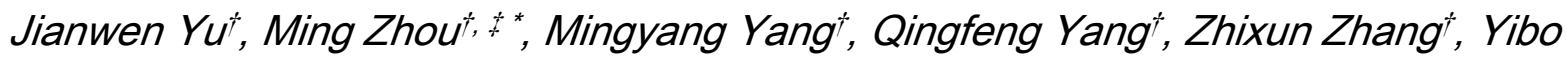

\section{Zhangt}

$\uparrow$ State Key Laboratory of Tribology, School of Mechanical Engineering, Tsinghua

University, Beijing 100084, China

$\$$ Key Laboratory for Advanced Materials Processing Technology, Ministry of Education,

Beijing 100084, China 

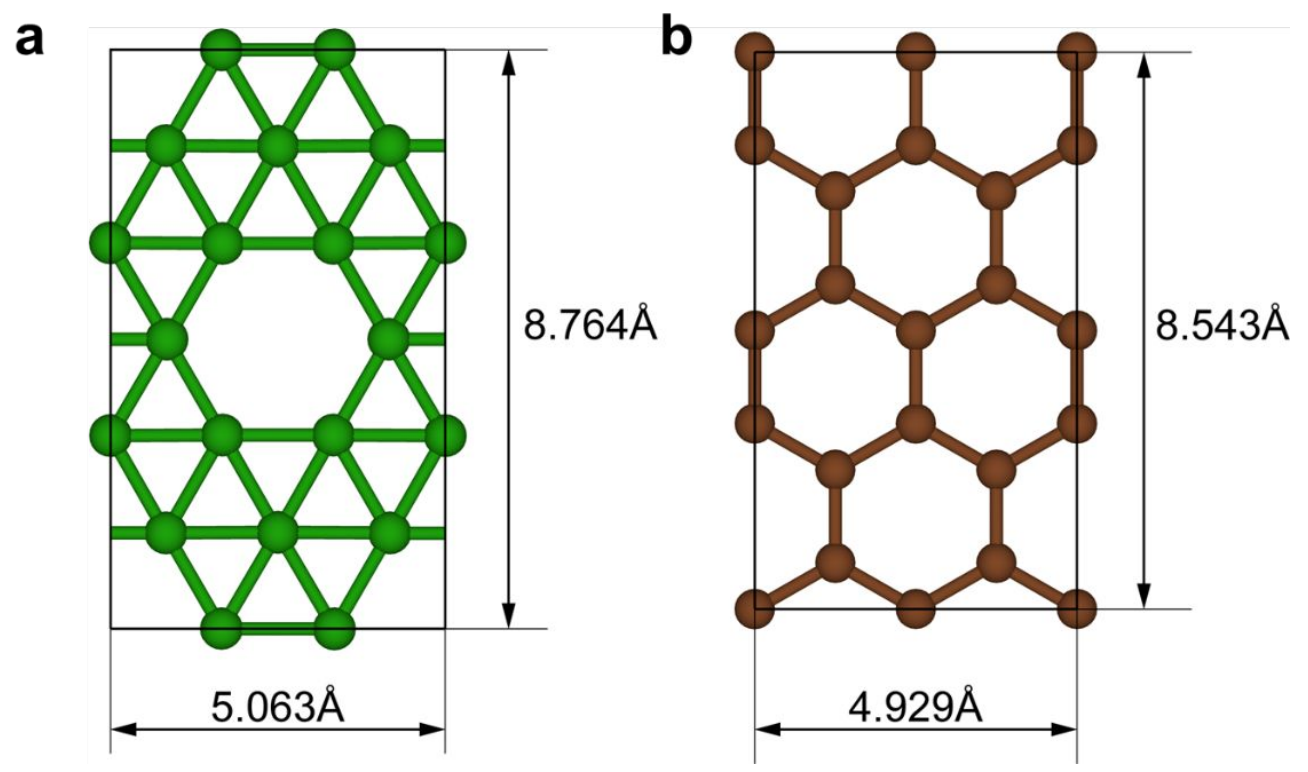

Figure S1. The optimized geometrical structures. (a) $\alpha$-sheet borophene and (b) graphene 


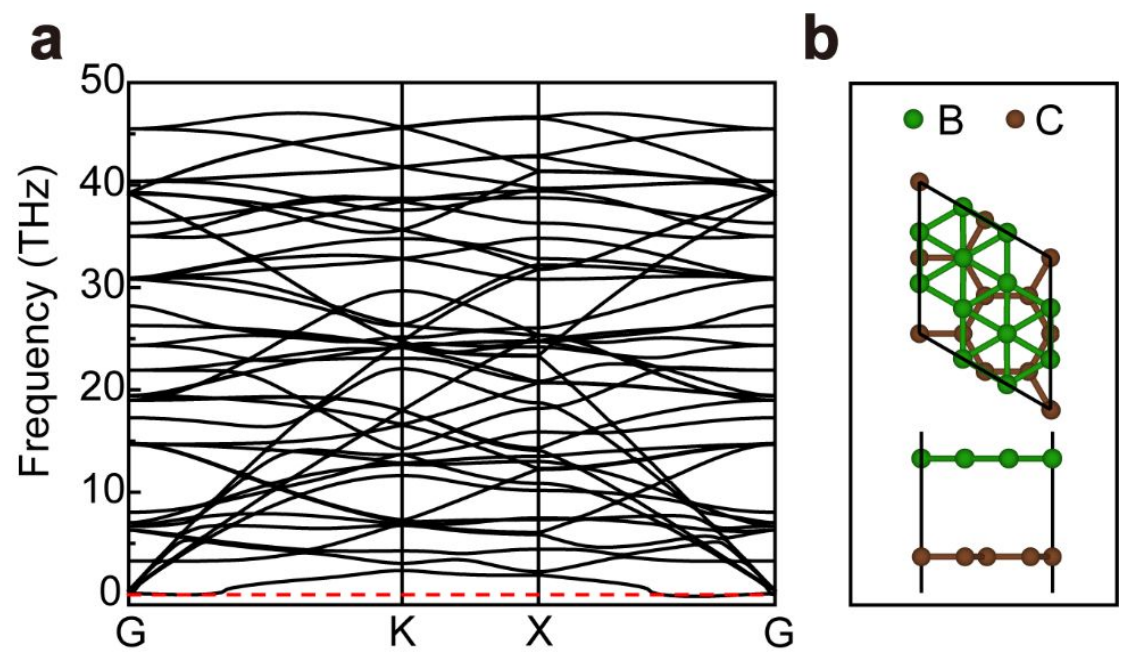

Figure S2. The phonon spectra of B/G. (a) The phonon spectra of B/G in (b) hexagonal cell. 


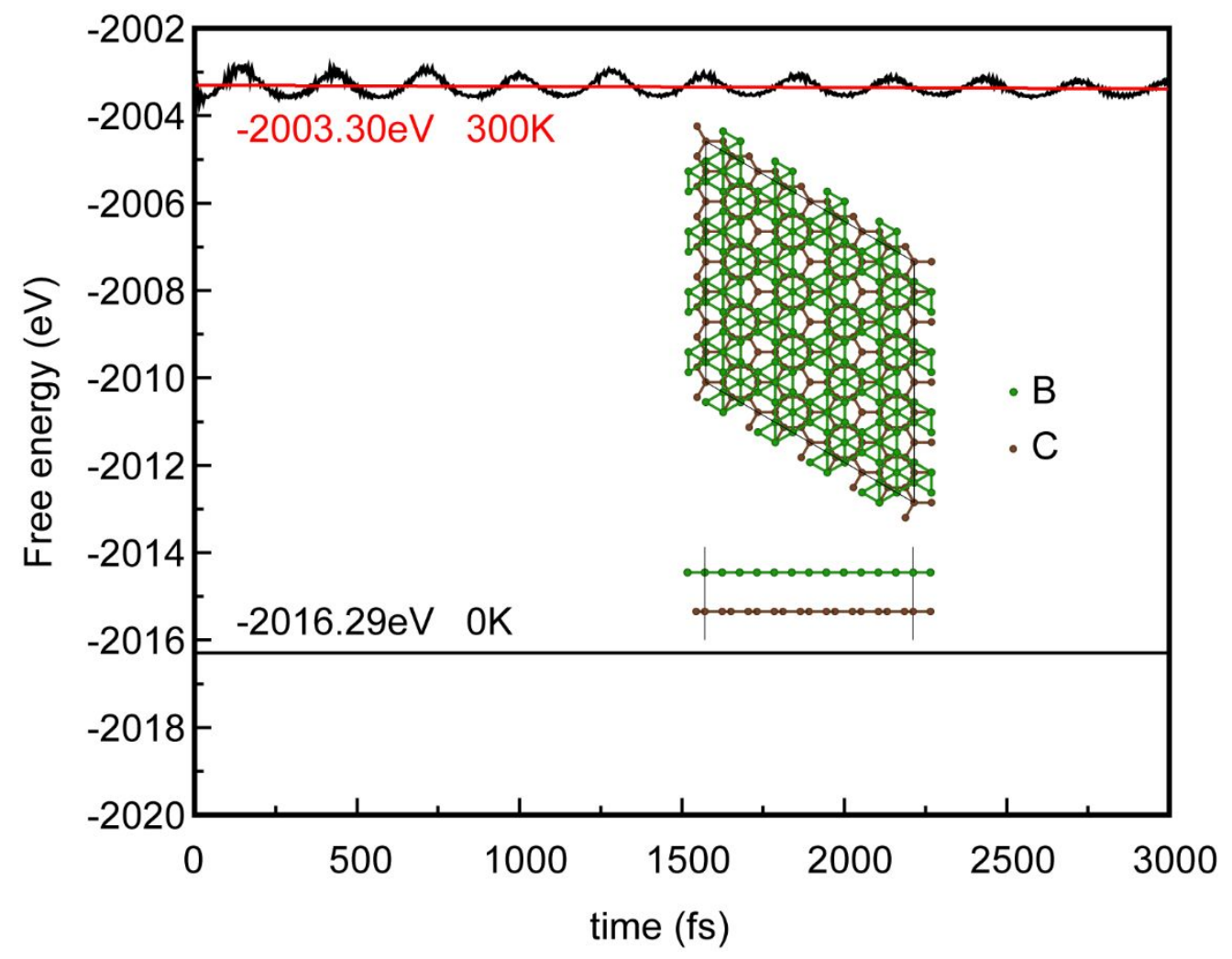

Figure S3. The free energy of $B / G$ by ab initio molecular dynamics (AIMD) in $300 \mathrm{~K}$, compared with using first-principles method. The red line is the trend line of energy fluctuation of $B / G$ in $300 K$. 

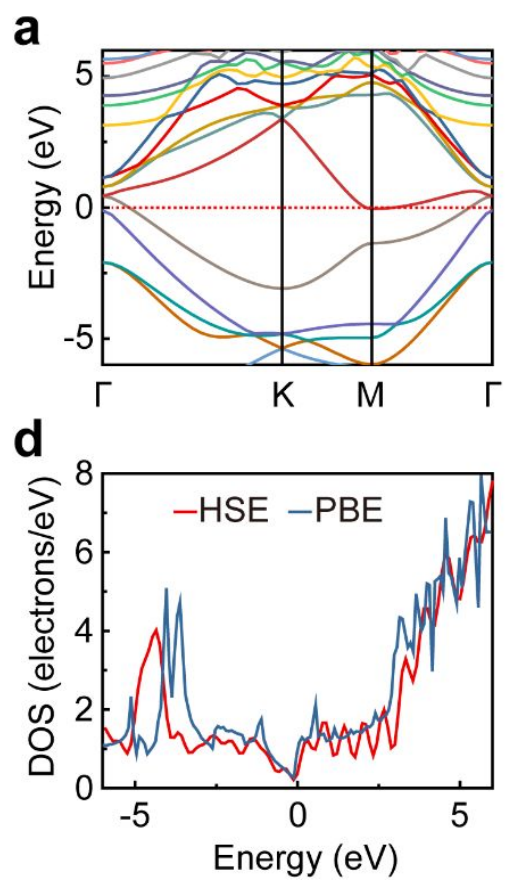
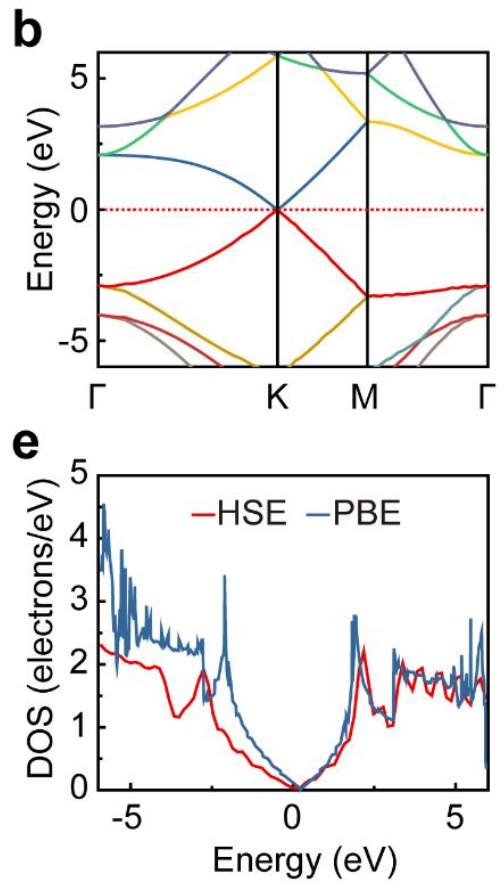
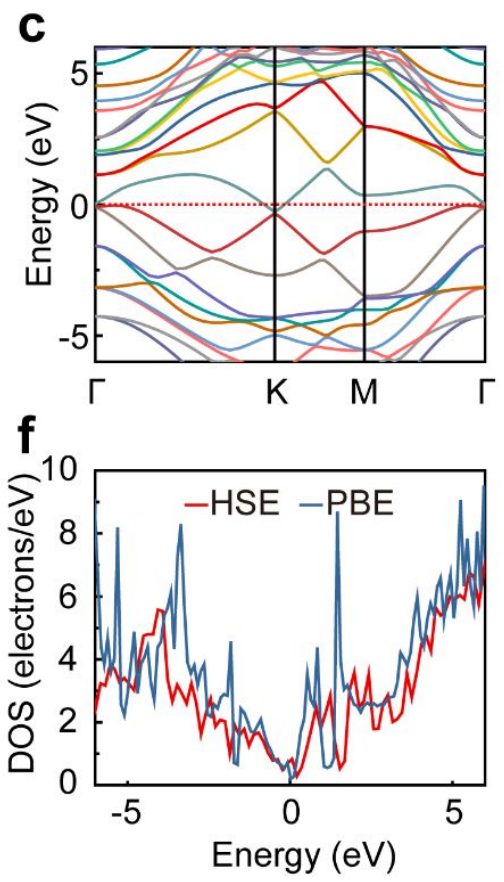

Figure S4. The band structures and density of states (DOS) of monolayer borophene, monolayer graphene and B/G in the hexagonal cell of Figure $S 2 b$. The band structures of (a) monolayer borophene, (b) monolayer graphene and (c) B/G by HSE. The DOS of (d) monolayer borophene, (e) monolayer graphene and (f) B/G by both PBE and HSE. There are few differences of DOS around Fermi level between HSE and PBE. 

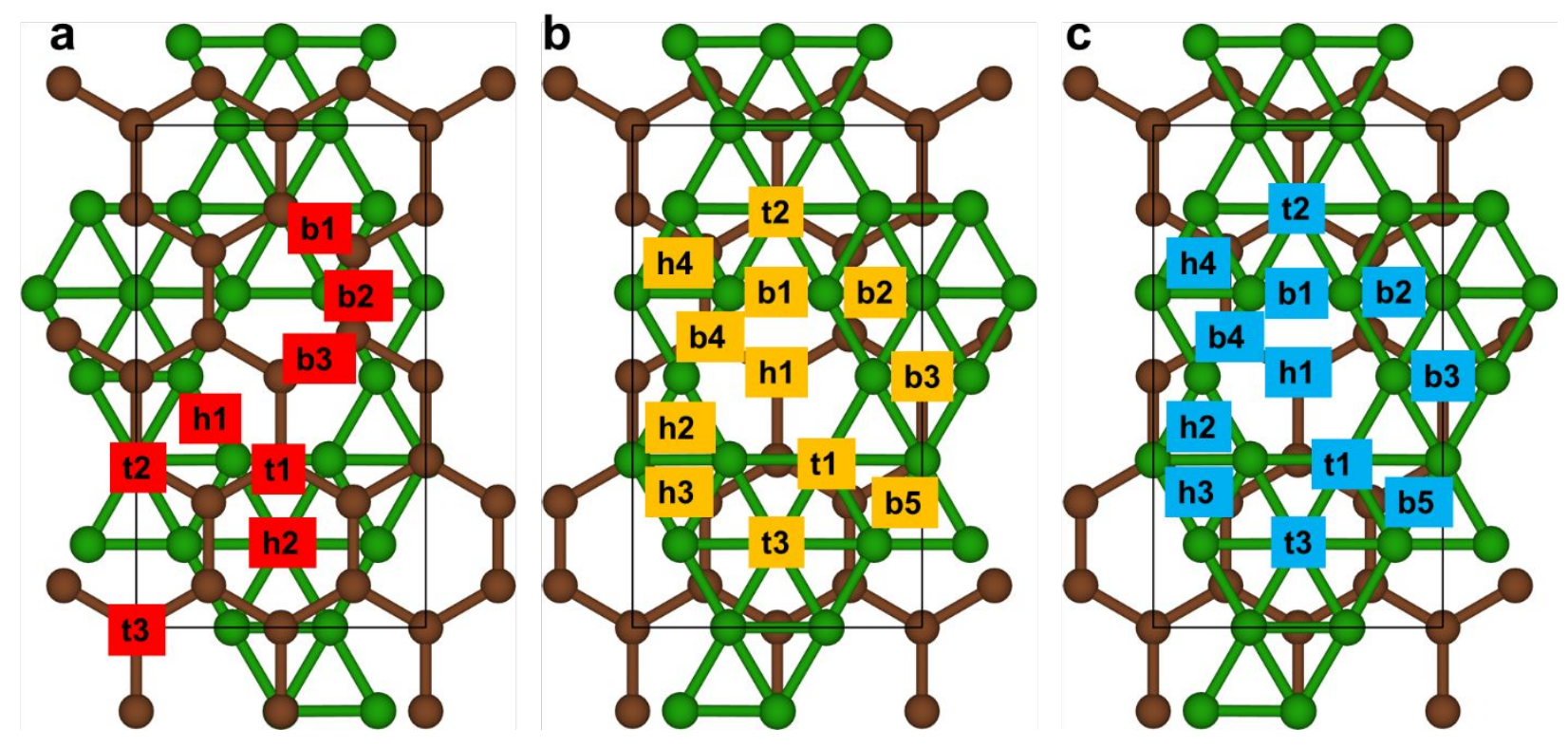

Figure S5. Possible adsorption sites of isolated Li on B/G heterostructure. (a) The sites outside of graphene in $B / G$, (b) in the interlayer of $B / G$ and (c) outside of borophene in B/G. Brown atoms for carbon, green atoms for boron. 

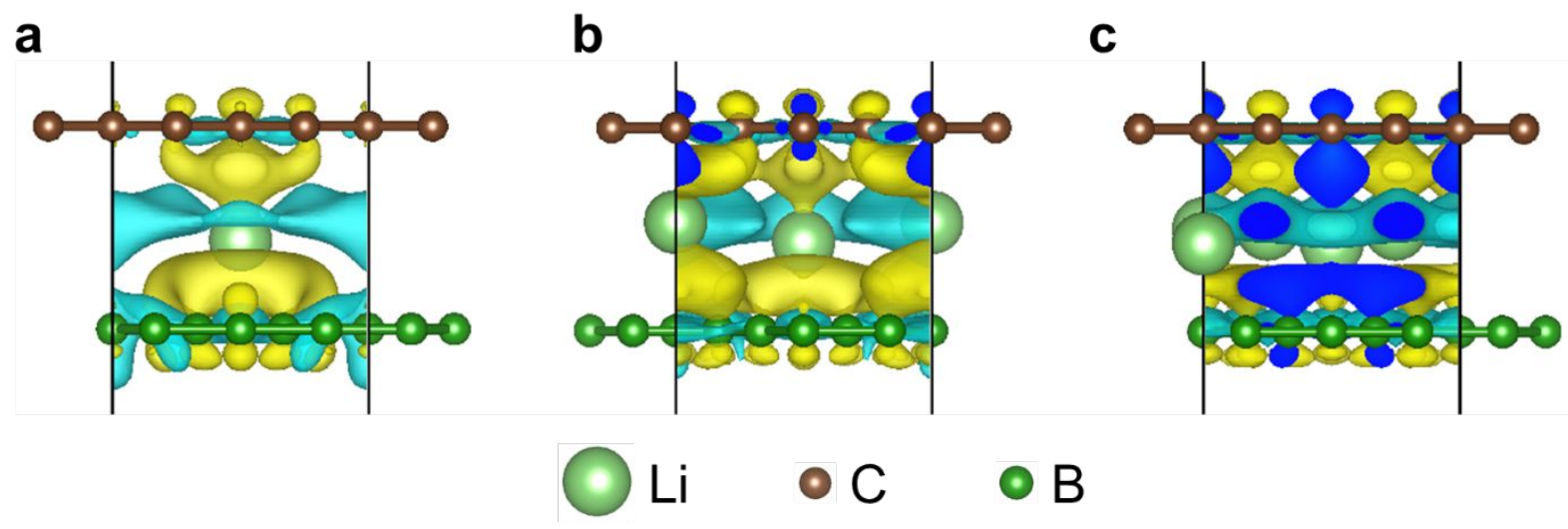

Figure S6. Deformation charge density of adsorption system. (a) $1 \mathrm{Li}$ with an isosurface value of $1.02 \times 10-3$ e/ $\AA$ 3 (b) 3 Li with an isosurface value of $2.06 \times 10-3$ e/ $\AA 3$ (c) 8 Li with an isosurface value of $3.66 \times 10-3$ e/ $\AA 3$ between borophene and graphene. The light blue and yellow regions indicated electron depletion and accumulation, respectively. Deep blue region was the cross section of the isosurface. 


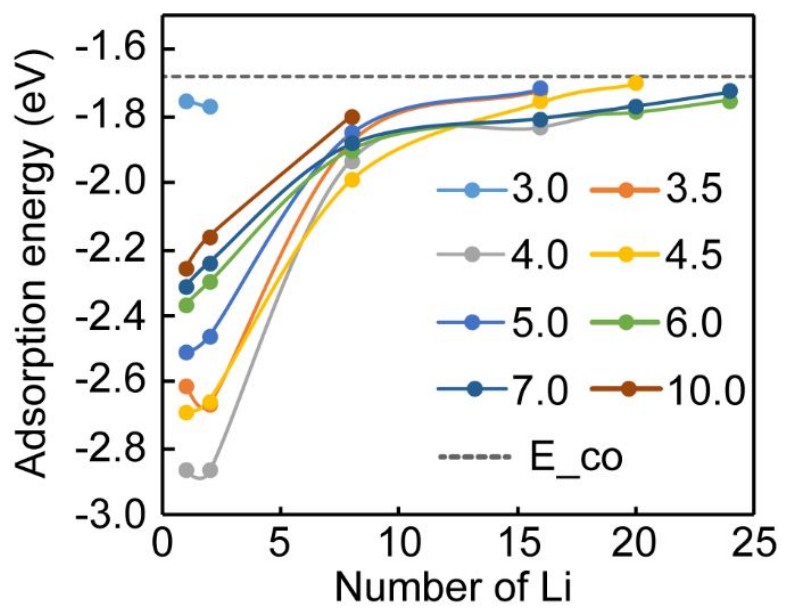

Figure S7. The relationship between the adsorption energy and the adsorption number of

$\mathrm{Li}$ at different interlayer distances. $\mathrm{E}_{-} \mathrm{Co}$ is the cohesive energy of Li metal, which is $1.67 \mathrm{eV}$. 


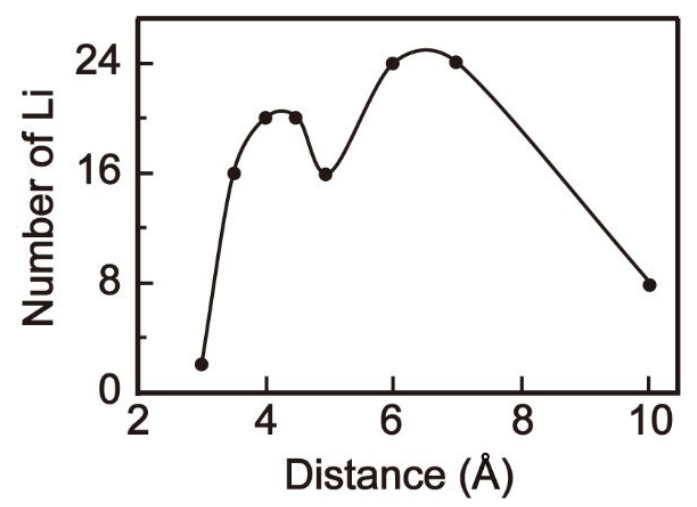

Figure S8. The relationship between the number of $\mathrm{Li}$ and the interlayer distance of $\mathrm{B} / \mathrm{G}$. 